\title{
Evaluation of computer generated neonatal discharge summaries
}

\author{
T Lissauer, C M Paterson, A Simons, R W Beard
}

\begin{abstract}
Computer generated and dictated discharge summaries were compared for all 133 babies admitted for intensive and special care during a six month period. Whereas 130/133 (98\%) had a computer generated summary, only $94 /$ $133(71 \%)$ had a dictated summary. In addition, computerised summaries were completed at discharge, but there was a delay up to 26 weeks for dictated summaries. Dictated summaries had more items of basic data missing but were more readable. A main diagnosis was missing in only $5 / 95(5 \%)$ of dictated and 1/130 (1\%) computerised summaries. Of the computer generated summaries, $114 / 133(86 \%)$ were suitable to give to parents.

Satisfactory discharge summaries for babies requiring intensive or special care can be generated with an on line computer system.
\end{abstract}

Continuity of care for babies discharged from neonatal units relies on good communication between hospital and community health professionals. Traditionally this has been achieved by doctors dictating discharge summaries, which are then typed and circulated. Compiling summaries for babies who have been in intensive care can be time consuming and arduous, especially when there have been several weeks between the baby's acute illness and his discharge from hospital. Selecting information without omitting important items is hampered by difficulty in finding the relevant information among copious notes, and the summary then needs to be typed, corrected, and distributed.

We have developed a neonatal information system into which data are entered on line by paediatricians who benefit from the automatically generated discharge summary. As the neonatal module is an integral part of an established maternity system-the St Mary's Maternity Information System-registration information and details of the pregnancy, delivery, and birth are transferred directly, avoiding re-entry of these data. ${ }^{12}$ The system also provides data for neonatal, obstetric, and administrative audit and generates ICD (International Classification of Diseases) codes for all neonates.

The St Mary's Maternity Information System is installed in all maternity units in the North West Thames Health Region, and covers $\mathbf{4 0} 000$ deliveries each year. The neonatal module was piloted in 1988 in the neonatal unit at St Mary's Hospital, and more recently in Hillingdon and Ealing hospitals.
We have conducted an initial evaluation of the computer generated summaries by comparing them with dictated summaries. We noted the number of summaries and the time it took to complete them, and compared a number of basic data items and the diagnoses for each baby. We also looked at their readability. As we planned to give a copy of the summary to the parents, we also assessed whether or not we considered the summaries suitable for them.

\section{Materials and methods}

DATA COLLECTION

Clinical data about any infant requiring intensive or special care are entered into the computer by paediatric staff who select relevant systems from a menu (table 1 ). To minimise the need to enter negative answers, a branching method of data entry is used so that further information is only requested when relevant, only systems with abnormalities are selected, and only important problems are entered.

For short stays in the baby unit all the clinical information can be entered when the baby leaves the unit. For complicated patients who stay a long time information is entered at least weekly to ensure its accuracy.

At discharge a list of diagnoses linked to ICD codes is generated by the computer. This is checked, amended as necessary, and the diagnoses ranked in order of importance by the paediatrician. In addition, advice on pertussis immunisation is entered and a statement is made about the baby's neurological and ophthalmological status at discharge. Free text can be added to the discharge letter; this is particularly useful for expanding social problems and discussing prognoses that do not lend themselves to computerisation, but it is discouraged for medical problems.

When data entry is complete the discharge summary is generated. Copies are printed for the neonatal notes, general practitioner, midwife or health visitor, or both, and the obstetric notes. A copy may also be printed for

Table 1 Systems menu

\section{Respiratory}

Birth asphyxia/trauma

Cardiovascular

Cardiovascular

Neurologica

Fluids, metabolic, and drug withdrawal

Nutrition, gastrointestinal, and bones

Haematology

Infection and temperature

Urogenital

Congenital abnormalities/syndromes and skin

Congenital ab

Additional comments

Infant death 
the mother. The data are also available for audit, and monthly and annual activity reports have been designed. The computer is also used by nursing staff to generate the daily bed state, which includes details of the level of care each baby is receiving.

\section{METHODS}

During the six month period from August 1988 to January 1989 when the computer system was being piloted, discharge summaries were dictated by the senior registrar using information from the clinical notes. These were then typed by a secretary who kept a record of the date the summary was typed, and filed a copy for the study. Concurrently data was entered on computer by the paediatric senior house officers on a terminal in the baby unit, and the summaries were generated when the babies were discharged. These were then also filed and kept for this study. They were not available when the summary was dictated nor were they distributed to general practitioners. Dictated summaries and those generated by computer were compared with the notes by a doctor (AS) with neonatal experience but who was not working in the neonatal unit during the study period, and therefore had no personal knowledge of the patients.

The following assessments were performed:

\section{The time of completion of summaries}

The summaries were matched with admissions to the unit to find out how many summaries were not completed.

\section{Basic data}

A number of items of information were identified that we considered should be included in all summaries (table 2). Each summary was checked to see how many items were missing.

\section{Accuracy of clinical data}

The notes were examined and a list of the main diagnoses was made for each baby. As no strict criteria for inclusion or exclusion could be made, personal clinical judgment was used to identify the most important diagnoses. These were checked against those listed in the dictated

Table 2 Items that should be included in a discharge summary

Registration information
Dates of admission and discharge
Reason for admission
List of diagnoses
Mode of delivery and reason for intervention
Apgar scores
Gestation and sex
Birth order if multiple birth
Birth weight and birth head circumference
Conditions requiring treatment
Congenital abnormalities
Treatment given in hospital
Treatment at discharge
Prognosis and residual disability at discharge
Immunisation recommendations and any immunisations given
Age at discharge or death
Discharge weight or head circumference
Feeding at discharge
Destination at discharge
Adverse social factors
Follow up with dates of appointments

and computerised summaries. In addition, the five most complex notes were selected and all items of data were listed and compared.

\section{Readability}

A measure of readability was made for each summary on a scale of 0 to 10 . Readability was judged according to how well the information about the baby could be assessed by reading through the summary. No strict criteria for this measure could be laid down.

\section{Suitability of summaries for parents}

An assessment was made of whether the computer summary was suitable for the parents. Summaries were deemed unsuitable if they were too technical or if it was thought that they might upset parents because the information was not imparted in a sensitive way.

\section{Results}

One hundred and thirty three infants were discharged or died between August 1988 and January 1989 and their notes and summaries were compared.

\section{Time of completion of summaries}

Whereas $130(98 \%)$ had a completed computer discharge summary only $94(71 \%)$ had a dictated summary. Computerised summaries were completed at the time of discharge from hospital, but the completion times for dictated summaries ranged from one to 26 weeks after discharge.

\section{Basic data}

Basic data that were missing or incorrect in the 94 dictated and 130 computer discharge summaries are shown in table 3 . Data relating to birth weight, mode of delivery, and Apgar

Table 3 Basic data omitted or incorrect in dictated and computerised summaries. Figures are expressed as number (\%)

\begin{tabular}{|c|c|c|}
\hline & $\begin{array}{l}\text { Dictated } \\
(n=94)\end{array}$ & $\begin{array}{l}\text { Computerised } \\
(n=130)\end{array}$ \\
\hline $\begin{array}{l}\text { Missing data in discharge summarie } \\
\text { Diagnoses } \\
\text { Birth weight } \\
\text { Gestational age } \\
\text { Head circumference at birth } \\
\text { Mode of delivery } \\
\text { Reason for intervention ( } n=42) \\
\text { Apgar scores } \\
\text { Reason for admission to special } \\
\text { care baby unit } \\
\text { Weight at discharge } \\
\text { Head circumference at discharge } \\
\text { Feeding at discharge } \\
\text { Discharge destination } \\
\text { Treatment on discharge }(n=36) \\
\text { Immunisation recommendations } \\
\text { Date of follow up appointment } \\
(n=47)\end{array}$ & $\begin{aligned} & 5(5) \\
& 33(35) \\
& 17(18) \\
& 85(90) \\
& 14(15) \\
& 2(5) \\
& 11(12) \\
& 3(3) \\
& 61(65) \\
& 70(75) \\
& 65(69) \\
& 63(67) \\
& 12(33) \\
& 19(20) \\
& 46(99)\end{aligned}$ & $\begin{array}{c}1(1) \\
0 \\
1(1) \\
11(9) \\
0 \\
39(93) \\
0 \\
2(2) \\
6(5) \\
13(10) \\
5(4) \\
3(2) \\
1(3) \\
6(5) \\
6(13)\end{array}$ \\
\hline Total & 506 & 94 \\
\hline $\begin{array}{l}\text { Errors in discharge summaries: } \\
\text { Sex } \\
\text { Mode of delivery } \\
\text { Birth weight }\end{array}$ & $\begin{array}{l}1(1) \\
6 \text { (6) } \\
2(2)\end{array}$ & $\begin{array}{l}\mathbf{0} \\
\mathbf{0} \\
\mathbf{0}\end{array}$ \\
\hline Total & 9 & 0 \\
\hline
\end{tabular}


scores are transferred from the maternity system and were complete in the computerised summaries. Details transferred from the obstetric system are partly validated at the time of entry, and a recent independent validation of all items included in the maternal discharge summaries during a three month period at this hospital showed an accuracy of over $99 \%$. As the reasons for operative delivery are often complex, they are not routinely recorded on either the obstetric or the neonatal computer. This meant that unless the reason for intervention was mentioned in the additional comments there was no record on the computerised summaries. Discharge information was often omitted from the dictated notes, and the babies' weight at discharge was omitted in 61 of the 94 $(65 \%)$ summaries as it was recorded in a different section of the babies' records. Whether pertussis immunisation was contraindicated was omitted from 19 of the 94 dictated summaries ( $20 \%$ ), compared with six of the 130 computerised summaries $(5 \%)$. In a number of the dictated summaries there were gross errors in the data. In one the baby's sex was incorrectly documented, in six the mode of delivery was wrong, and in two the birth weights were wrong.

In 21 of the dictated summaries there was no indication either by name or pronoun of the sex of the infant.

\section{Accuracy of clinical data}

Five of the diagnoses were missing in the 94 dictated summaries and one in the 130 computerised summaries.

\section{Readability}

Readability scores showed that the dictated summaries were more readable, with a median score of 8 , compared with 6 for the computer generated summaries.

The dictated summaries gave a more complete impression of the events leading up to birth, the sequence of events in the neonatal period, and the long term prognosis especially of preterm infants with long, complex medical histories. This difference was less obvious when paediatricians had used the facility to add additional comments to the computerised summary. In the 130 summaries reviewed, $28(22 \%)$ had additional comments entered, $52(40 \%)$ would have been improved if additional comments had been entered, and $50(38 \%)$ summaries did not require any further information. It was thought that the computerised summaries would also have been easier to read with a better quality printer. Although dictated summaries tended to list events in chronological order, which made them easier to follow, it was harder to find specific information without reading the entire summary.

\section{Suitability of summaries for parents}

It was thought that the computerised summary was suitable in its present form for $114(88 \%)$ of infants. They were considered unsuitable for parents when the babies were discharged to foster parents as they contained too many maternal details. The summaries are rather technical and brusque and it was thought that the lack of sympathy could distress parents of the nine babies who died, or the four with major chromosomal abnormalities.

\section{Discussion}

The primary objective of the design of the system was to produce a summary containing all the important information required when the baby left hospital. The summary should contain an accurate account of the baby's major problems in hospital, and any items of relevance to long term care. Whereas virtually all $(98 \%)$ of the computer generated summaries were done at discharge only $71 \%$ of babies admitted to the baby unit had a dictated summary with delays of up to 26 weeks. As well as depending on the diligence of the registrar, delay sometimes occurred because the baby was discharged from the postnatal wards and by the time the notes had been returned to the neonatal unit for dictation they were needed in clinics. Holidays, study leave, and change of staff further exacerbated the delay. Had the summaries been dictated by the senior house officers it is likely that more summaries would have been completed and some units do demand, and achieve, typewritten summaries before discharge. Dictating summaries for babies who have undergone intensive care is time consuming, however, and at busy times it is often difficult to do this before discharge.

The superiority of the computer in recording basic data at birth and discharge was readily apparent. In 21 of 94 (22\%) dictated summaries there was no indication of the sex of the infant. Computers can enforce greater completeness of data, and can be programmed to ensure that advice on routine procedures such as pertussis immunisation is included.

The lists of diagnoses generated by the computer were slightly more complete than those in the dictated summaries. Listing the diagnoses, linked to ICD codes, in order of priority enables the system to comply with Department of Health requirements.

No attempt was made to check in detail the accuracy of the data entered by the paediatrician. Only information of clinical importance should be entered and this depended on the discretion of the person entering the data; each item of data needs to be carefully defined. In addition, the reliability of the data collected needs to be checked-for example, for babies receiving artificial ventilation several paediatricians have requested that the maximum inspired oxygen concentration, peak ventilatory pressure, and maximum ventilatory rate be recorded, but we have found that these data cannot be collected accurately. Should they be the highest ever employed, or should they refer to values once the baby is stabilised? Further development is required to decide which items of data should be collected and which can be dispensed with.

Dictated summaries were usually more read- 
able and gave a chronological sequence of events. They also provided more comprehensive information about social circumstances and prognosis. They often contained much superfluous information, however, and it is possible that long and detailed summaries are not always read by the health professionals for whom they are intended. Computerised summaries organised by system are less readable, but specific items of information are easier to find. Additional comments in free text are clearly important and the facility was underused. Usually only one or two sentences are required. We consider that the computer generated summaries are now sufficiently well developed for distribution to general practitioners and other health professionals for formal assessment of their usefulness.

One advantage of computerised summaries is that it is easy to give a copy to the mother before she leaves hospital and it can be used to initiate or supplement the baby's personal held record. We considered that most of the summaries were suitable for parents but all summaries need to be carefully checked. We have not yet sought the views of parents and it may be that we have been overprotective. Particular care needs to be taken when babies have died, have a major congenital abnormality, experienced birth asphyxia or intraventricular haemorrhage, or when they have been born to drug users, to make sure that the summaries correspond with what parents know about their baby and cannot cause confusion or offence, or lead to litigation. Even when the computerised summary is unsuitable for parents it can be used to provide the basic facts for a dictated summary.

As a result of this pilot study, the computer generated summaries have been improved and in particular have been made more readable. We believe that they now compare even more favourably with dictated summaries. Since the completion of this study the computer generated summaries have been successfully introduced into routine use.

1 Maresh M, Beard RW, Dawson AM, et al. Selection of an obstetric data base for a microcomputer and its use for on-line production of birth notification forms, discharge summaries and perinatal audit. Br F Obstet Gynaecol 1983, 90:227-31.

2 Maresh M, Dawson AM, Beard RW. Assessment of an online computerised perinatal data collection and information system. Br f Obstet Gymaecol 1986;93:1239-45. 